\title{
A novel compressive sampling system for chirp signal
}

\author{
Enpin Yanga), Xiao Yan, and Kaiyu Qin \\ School of Aeronautics and Astronautics, University of Electronic Science and \\ Technology of China, \\ No. 2006 Xiyuan Ave, West Hi-Tech Zone, Chengdu 611731, China
}

a)yang_enpin@163.com

\begin{abstract}
In this letter, a compressive sampling system, which can acquire chirp signal without the prior information of carrier frequency and chirp rate, is proposed. The system first estimates the chirp rate based on windowed modulated wideband converter (MWC). When chirp rate is derived, the system can de-chirp the target signal. Then, the de-chirped signal is acquired by sampling module of the system. Finally, basedon samples, the original signal can be reconstructed by a greedy iteration algorithm which has an adaptive halting condition. Simulation results show the system is capable of estimating chirp rate and reconstructing original signal.
\end{abstract}

Keywords: compressive sampling, chirp signal, MWC, chirp rate estimation, signal reconstruction

Classification: Circuits and modules for electronic instrumentation

\section{References}

[1] D. Zhu, et al.: "A novel high-precision phase-derived-range method for direct sampling LFM radar," IEEE Trans. Geosci. Remote Sens. 54 (2016) 1131 (DOI: 10.1109/TGRS.2015.2474144).

[2] F. Guo, et al:: "TDOA/FDOA estimation method based on dechirp," IET Signal Process. 10 (2016) 486 (DOI: 10.1049/iet-spr.2015.0460).

[3] R. Middleton: "Dechirp-on-receive linearly frequency modulated radar as a matched-filter detector," IEEE Trans. Aerosp. Electron. Syst. 48 (2012) 2716 (DOI: 10.1109/TAES.2012.6237622).

[4] C. Parkey, et al.: "Linearized adaptation of non-linear post conversion correction for TIADCs: a behavioral model study," IEEE Instrum. Meas. Mag. 18 (2015) 46 (DOI: 10.1109/MIM.2015.7155773).

[5] A. Salimath, et al:: "A 6 bit $800 \mathrm{MHz}$ TIADC based on successive approximation in $65 \mathrm{~nm}$ standard cmos process," ICVD (2010) 312 (DOI: 10.1109/VLSI.Design.2010.55).

[6] A. Bonnetat, et al: "An adaptive all-digital blind compensation of dual-TIADC frequency-response mismatch based on complex signal correlations," IEEE Trans. Circuits Syst. II, Exp. Briefs 62 (2015) 821 (DOI: 10.1109/TCSII.2015. 2435611).

[7] M. Mishali and Y. C. Eldar: "Xampling: signal acquisition and processing in union of subspaces," IEEE Trans. Signal Process. 59 (2011) 4719 (DOI: 10. 1109/TSP.2011.2161472). 
[8] Y. Lu and M. Do: "A theory for sampling signals from a union of subspaces," IEEE Trans. Signal Process. 56 (2008) 2334 (DOI: 10.1109/TSP.2007.914346).

[9] M. Unser: "Sampling: 50 years after shannon," Proc. IEEE 88 (2000) 569 (DOI: 10.1109/5.843002).

[10] E. Candes and T. Tao: "Near optimal signal recovery from random projections: universal encoding strategies," IEEE Trans. Inf. Theory 52 (2006) 5406 (DOI: 10.1109/TIT.2006.885507).

[11] D. Donoho: "Compressed sensing," IEEE Trans. Inf. Theory 52 (2006) 1289 (DOI: 10.1109/TIT.2006.871582).

[12] M. Mishali and Y. C. Eldar: "From theory to practice: sub-nyquist sampling of sparse wideband analog signals,” IEEE J. Sel. Topics Signal Process. 4 (2010) 375 (DOI: 10.1109/JSTSP.2010.2042414).

[13] M. Mishali and Y. C. Eldar: "Blind multiband signal reconstruction: compressed sensing for analog signals," IEEE Trans. Signal Process. 57 (2009) 993 (DOI: 10.1109/TSP.2009.2012791).

[14] M. Mishali and Y. C. Eldar: "Wideband spectrum sensing at sub-nyquist rates," IEEE Signal Process. Mag. 28 (2011) 102 (DOI: 10.1109/MSP.2011.941094).

[15] E. Yang, et al:: "Modulated wideband converter with run length limited sequences," IEICE Electron. Express 13 (2016) 20160670 (DOI: 10.1587/elex. 13.20160670).

[16] E. Yang, et al:: "A greedy pursuit algorithm for arbitrary block sparse signal recovery,” ISCAS (2016) 1234 (DOI: 10.1109/ISCAS.2016.7527470).

[17] E. Yang, et al.: "Arbitrary block sparse signal reconstruction based on incomplete single measurement vector," Circuits Syst. Signal Process. (2017) accepted (DOI: 10.1007/s00034-017-0528-3).

\section{Introduction}

The chirp signal is generated by linear frequency modulation (LFM) which can be formulated as $x(t)=z(t) \cos \left(2 \pi f_{0} t+\pi k t^{2}\right)$ (Here, $z(t)$ is the baseband signal, $f_{0}$ is the carrier frequency and $k$ is the chirp rate), and is widely used in many applications, such as LFM radar, sonar, communication and real-time spectrum analysis. Since the spectrum of chirp signal has an ultra-wide band, most of the sampling systems require the prior information of carrier frequency and chirp rate, otherwise they cannot acquire the target signal $[1,2,3]$. When these information are known, those sampling systems can first de-chirp the target signal to reduce its bandwidth (the de-chirped signal is formulated as $x(t)=z(t) \cos \left(2 \pi f_{0} t\right)$ which is always a narrow band signal), then based on the carrier frequency, the de-chirped signal can be acquired at a low rate by Nyquist sampling or bandpass sampling. When the prior information is unavailable, a conventional method of acquiring the chirp signal is to use an ultra-high speed sampling system which satisfies the wellknown Nyquist sampling theorem, for example, the time-interleave analog-todigital converter (TIADC) $[4,5,6]$. However, the Nyquist sampling theorem is inefficient in many applications, because samples produced by an ultra-high speed sampling system are extremely redundant $[7,8,9]$. Obviously, acquiring the chirp signal efficiently without any prior information is a tough task, which is required in many applications, such as cognitive radio, communication monitoring and realtime spectrum analysis. 
Recent years, some compressive sampling (CS) methods [10, 11], which only need a relatively smaller sampling rate compared with these methods which conform to the Nyquist theorem, have attracted increasing attention. Within the CS framework, the modulated wideband converter (MWC) is the most practical system $[12,13,14,15]$. The MWC is designed for processing multiband signals (if all spectral contents of a signal reside within a few disjoint frequency intervals, then the signal is called multiband signal [12]). It scrambles the spectrum of multiband signal via modulation, then all spectral contents can be transferred to a low-frequency band, after low-pass filtered the scrambled signal can be sampled at a low rate, finally, the original signal can be reconstructed based on these samples. Since spectral contents of a chirp signal reside within a large frequency range, it is obvious that the chirp signal is not a multiband signal, thus the MWC cannot deal with the chirp signal. Therefore, compressive sampling system for acquiring chirp signals should be investigated.

In this letter, we present an universal chirp signal compressive sampling system. The most advantage of our sampling system is that it does not require the prior information of carrier frequency and chirp rate of target signal. In the system, our main contribution is a chirp rate estimation algorithm and its theoretical condition that constrains the estimation range of chirp rate. The chirp rate estimation algorithm is designed by introducing window function into the MWC. Similar with the principle of short-time Fourier transform (STFT), we leverage the window function to obtain several short-time chirp signals which can be treated as multiband signals. When processing these short-time signals, the estimation algorithm can detect how frequencies change in different windows. Based on the frequencies variation information, chirp rate can be estimated. Both the theoretical analysis and simulation results show this algorithm is capable of estimating the chirp rate, as long as the rate satisfies the constraint condition. When the chirp rate is derived (the carrier frequency is still unknown), the system can de-chirp the target signal to convert it into a multiband signal, then the multiband signal can be sampled by the MWC. Note that although the de-chirped signal is processed by MWC, the signal reconstruction algorithm adopted by our system differs from that of the original MWC. Our another contribution is the signal reconstruction algorithm, which evolves from our previous work [16, 17]: the block matching pursuit (BMP) algorithm, and it is named multiple measurements BMP (MBMP) algorithm. The proposed reconstruction algorithm guarantees an outstanding reconstruction performance even if the chirp rate is not exactly estimated. Compared with the BMP in $[16,17]$, the most creative feature of the algorithm in this letter is the adaptive halting condition which makes the sampling system more practical.

\section{The chirp rate estimation algorithm}

Diagram of the proposed compressive sampling system is depicted in Fig. 1 (the sampling module of our system is the same with that of MWC). As mentioned in introduction and illustrated in Fig. 2(a) and Fig. 2(b), we utilize window function to truncate the input chirp signal, and based on several truncated signals, the chirp rate can be estimated. 


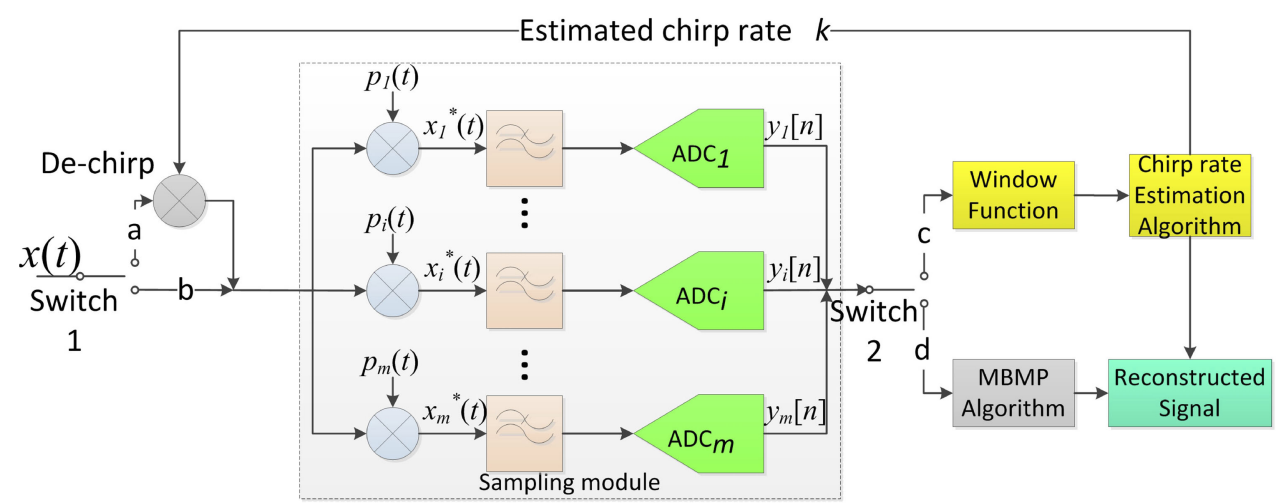

Fig. 1. Diagram of the compressive sampling system. In the beginning, Switch 1 connects with $\mathrm{b}$ and Switch 2 connects with $\mathrm{c}$. When the chirp rate is estimated, Switch 1 switches on a and Switch 2 switches on $\mathrm{d}$.

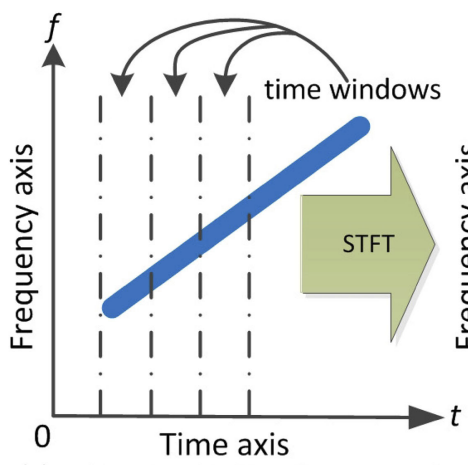

(a) a chirp signal in time-frequency plane

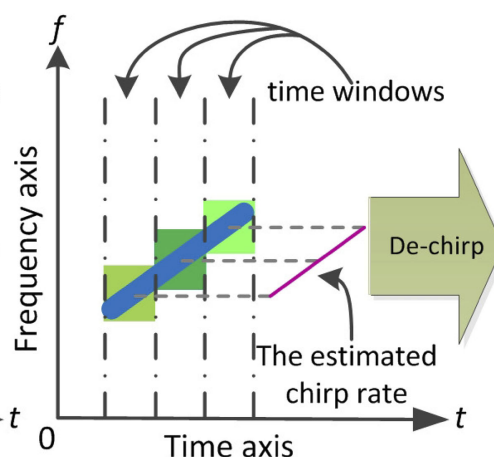

(b) STFT of the chirp signal

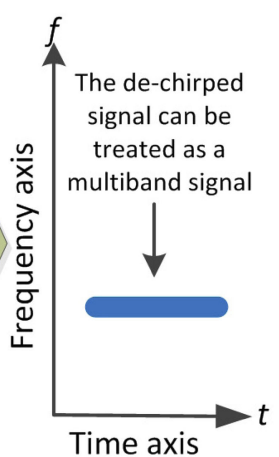

(c) the de-chirped signal

Fig. 2. How the system works. It first estimates the chirp rate, then dechirps the signal to obtain a multiband signal. The blue line indicates a chirp signal and these three green rectangles denote STFT.

When estimating the chirp rate, the chirp signal $x(t)$ is directly input into the sampling stage, which is controlled by Switch 1 . In the $i$-th channel $(1 \leq i \leq m$ and $m$ is the total channel number), the input signal is modulated by a sequence $p_{i}(t)$ with period $T_{p}$ [15]. Since $p_{i}(t)$ is periodic, it has a Fourier expansion

$$
p_{i}(t)=\sum_{l=-\infty}^{+\infty} c_{i l} e^{j \frac{2 \pi}{T_{p}} l t}, \quad c_{i l}=\frac{1}{T_{p}} \int_{0}^{T_{p}} p_{i}(t) e^{-j \frac{2 \pi}{T_{p}} l t} d t
$$

The short-time Fourier transform (STFT) of modulated signal $x_{i}^{*}(t)$ is

$$
\begin{aligned}
& \operatorname{STFT}_{x i}(t, f)=\int_{-\infty}^{+\infty} x_{i}^{*}(\tau) g(\tau-t) e^{-j 2 \pi f \tau} d \tau \\
& \quad=\sum_{l=-\infty}^{+\infty} c_{i l} \int_{-\infty}^{+\infty} x(\tau) g(\tau-t) e^{-j 2 \pi\left(f-\frac{l}{T_{p}}\right) \tau} d \tau=\sum_{l=-\infty}^{+\infty} c_{i l} \operatorname{STFT}_{x}\left(t, f-l f_{p}\right)
\end{aligned}
$$

where $f \in B$ ( $B$ is the input bandwidth of the system), $f_{p}=1 / T_{p}, g(\tau)$ is a window function (window width is determined by $\tau$ ) and $(\tau-t)$ means time shift. The function of modulation stage is to scramble the original spectrum in a single time window such that all spectral contents can be transferred to a low-frequency range 
[15]. After low-pass filtered (cutoff frequency is $1 / 2 T_{s}$ ) and sampled (sampling period $T_{s}=T_{p}$ ), based on [12], the STFT of samples can be derived

$$
\operatorname{STFT}_{y i}(t, f)=\sum_{l=-\infty}^{+\infty} y_{i}[n] g[\tau-t] e^{-j 2 \pi f n T_{s}}=\sum_{l=-L_{0}}^{+L_{0}} c_{i l} \operatorname{STFT}_{x}\left(t, f-l f_{p}\right)
$$

where $y_{i}[n]$ is samples of the $i$-th $\mathrm{ADC}, L_{0}$ is a positive integer which guarantees that all spectral contents can be transferred into the cutoff frequency range of filter and $L_{0}=\left\lceil B \times T_{S}\right\rceil(\lceil z\rceil$ returns the greater (or equal) integer which is closest to $z$; we refer the reader to [12] for details of $L_{0}$ ).

According to (3), STFT of samples of the $i$-th channel (i.e. $\operatorname{STFT}_{y i}(t, f)$ ) is a linear combination of $f_{p}$-shifted copies of STFT of the input signal (i.e. $\left.\operatorname{STFT}_{x}(t, f)\right)$. When taking all channels into account, (3) can be rewritten in the matrix form

$$
\boldsymbol{Y}(t, f)=\boldsymbol{A} \boldsymbol{X}(t, f)
$$

where $\boldsymbol{Y}(t, f)$ is a matrix whose $i$-th row vector is the STFT of $y_{i}[n], \boldsymbol{A}$ is an $m \times\left(2 L_{0}+1\right)$ matrix $\left(m<\left(2 L_{0}+1\right)\right)$ whose $i$-th row vector is the Fourier series of $p_{i}(t), \boldsymbol{X}(t, f)$ is a matrix which is the STFT of $x(t)$ (note that each column vector of $\boldsymbol{X}(t, f)$ is the spectrum of a short time signal truncated by one window and the row number of $\boldsymbol{X}(t, f)$ is $\left.\left(2 L_{0}+1\right)\right)$. When the input signal is a chirp signal, a diagram of $\boldsymbol{X}(t, f)$ is depicted in Fig. 3, here, each square denotes an entry of $\boldsymbol{X}(t, f)$ (white square means zero and colored square means nonzero spectral contents).

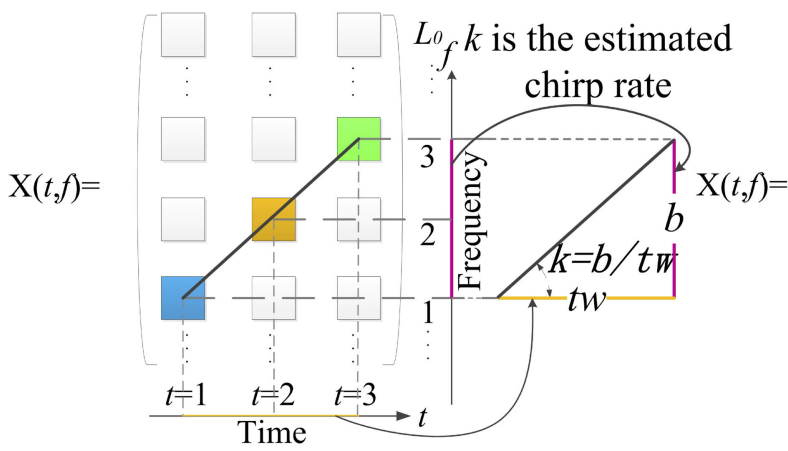

(a) When chirp rate is low

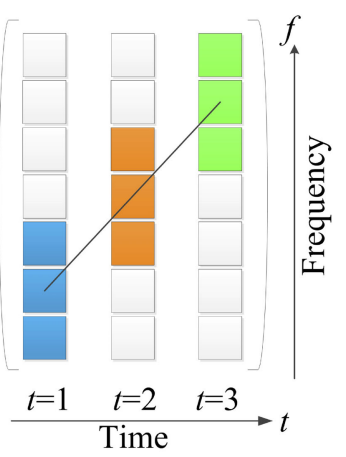

(b) When chirp rate is high

Fig. 3. Diagram of $\boldsymbol{X}(t, f)$ (two forms are depicted).

The chirp rate is estimated based on $\boldsymbol{X}(t, f)$. Then, we explain how to estimate it. As illustrated in Fig. 2(b) and Fig. 3, each entry of the matrix $\boldsymbol{X}(t, f)$ indicates a certain frequency bandwidth and time width. Since the frequency of chirp rate is time-varying, spectral support of signals (i.e. locations of spectral contents) is also varying in different time windows. When the $\boldsymbol{X}(t, f)$ is derived, the information of spectral support in different time windows is available, then the chirp rate $k$ is

$$
k=\frac{1}{w n-1} \sum_{i=1}^{w n-1} \frac{f_{i+1}-f_{i}}{t w}
$$

where $w n$ is the number of windows, $t w$ is the width of one window (all windows have the same width) and $f_{i}$ is the center frequency of spectral content in the $i$-th 
window. In (5), an average number is calculated to improve the accuracy and simulation results show $w n=3$ is adequate for estimating the chirp rate (see Section 4). Once the $\boldsymbol{X}(t, f)$ is derived, the chirp rate can be obtained. Therefore, (4) must be solved first. Within compressive sampling framework, only when $\boldsymbol{X}(t, f)$ is sparse (namely, it only contains a few nonzero rows), (4) can be solved. However, when processing chirp signal, $\boldsymbol{X}(t, f)$ may be not sparse. Thus, we convert the matrix $\boldsymbol{X}(t, f)$ into a column vector by concatenating rows of $\boldsymbol{X}(t, f)$, then the vector must be sparse since it only has a few nonzero rows. Namely, we solve (6) instead of (4)

$$
\operatorname{vec}(\boldsymbol{Y}(t, f))^{\prime}=(\boldsymbol{A} \otimes \boldsymbol{E}) \operatorname{vec}(\boldsymbol{X}(t, f))^{\prime}, f \in B
$$

where vec $(\boldsymbol{u})$ means concatenating all rows of matrix $\boldsymbol{u}, \operatorname{vec}(\boldsymbol{u})^{\prime}$ is column vector, $\boldsymbol{A} \otimes \boldsymbol{E}$ is the Kronecker product of $\boldsymbol{A}$ and $\boldsymbol{E}, \boldsymbol{E}$ is a $d$ dimension unit matrix and $d$ is the column number of $\boldsymbol{X}(t, f)$. The chirp rate estimation algorithm is summarized in Table I.

Table I. Pseudo-code of the chirp rate estimation algorithm

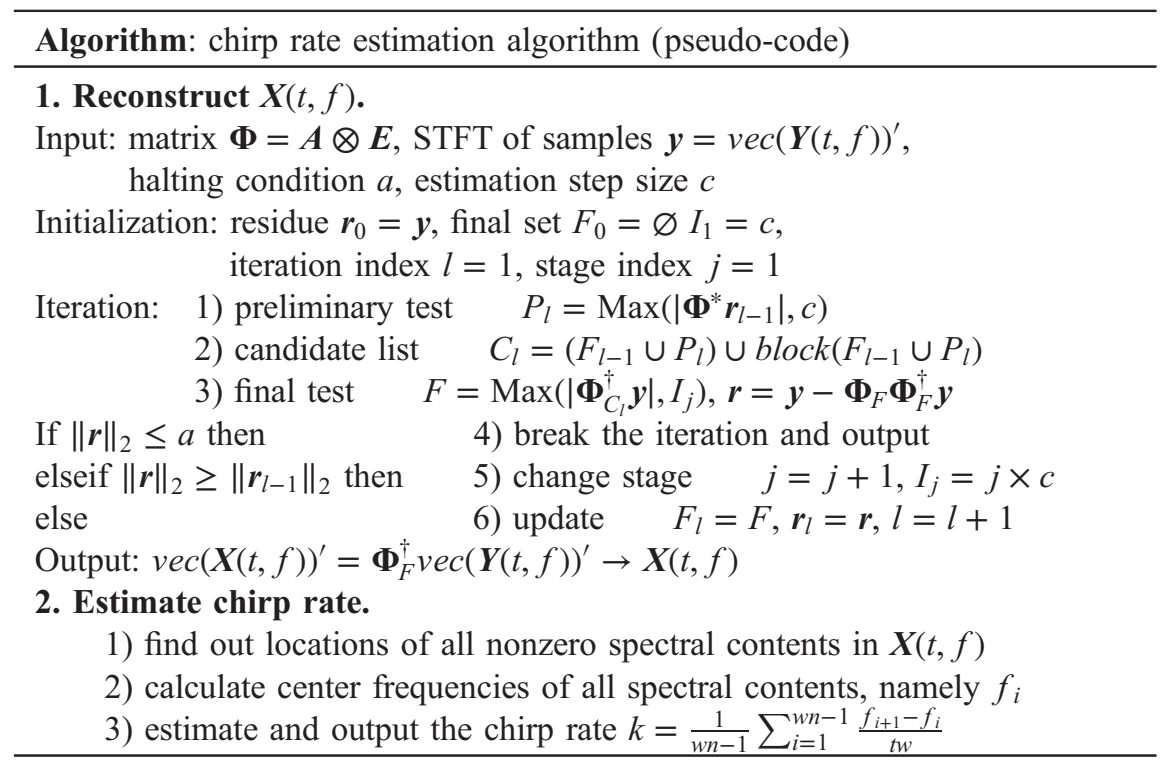

The $\boldsymbol{X}(t, f)$ reconstruction part of the algorithm is the BMP algorithm [16, 17], which is a greedy iteration algorithm and adopts a stagewise strategy to reconstruct the signal. The details of this part are introduced in the next section, because this part is similar with the MBMP algorithm in Section 3. We also refer the reader to our previous work $[16,17]$ for details of BMP.

In the following context, we develop the theoretical constraint of the chirp rate estimation algorithm. In Fig. 3(a), there is only one spectral content in each window. In this situation, the chirp rate is low. But if the chirp rate is very high, then $\boldsymbol{X}(t, f)$ is in the form of Fig. 3(b), and the number of spectral contents in a single window is relatively bigger. Conventionally, within compressive sampling framework, the number of spectral contents in a single window cannot exceed $m / 2$ ( $m$ is the row dimension of samples, in our case it is the number of sampling channel). Since our system is designed for sampling analog real signal whose 
spectrum has conjugation, the number of spectral contents in a single window cannot exceed $m / 4$. Then, the occupied bandwidth of all spectral contents in a single time window has an upper bound

$$
\max (\text { bandwidth })=\frac{m}{4} \times \frac{B}{2 L_{0}+1}
$$

where $m / 4$ is the maximum number of spectral contents in a single window and $B /\left(2 L_{0}+1\right)$ is the bandwidth of a spectral content ( $B$ is the input bandwidth of the system). Furthermore, in each time window, the number of samples in one time window must be greater than $m / 2$ to ensure the rank of $\boldsymbol{Y}(t, f)$ is not smaller than the number of spectral contents, otherwise, there is no solution for (6) (i.e. no solution for (4)). Then, the smallest width of a time window is

$$
\min (\text { Time_width })=\frac{m}{2} \times T_{s}
$$

where $T_{s}$ is the sampling period. The chirp rate equals the bandwidth in a period of time divided by time. Therefore, as long as the chirp rate $k$ satisfies (9), it can be estimated.

$$
-\frac{\max (\text { bandwidth })}{\min (\text { Time_width })}<k<\frac{\max (\text { bandwidth })}{\min (\text { Time_width })}
$$

\section{The signal reconstruction algorithm}

When the chirp rate $k$ is derived, the input signal is de-chirped before entering the sampling stage, which is controlled by Switch 1. First, we explain how to de-chirp the signal by (10)

$$
\begin{aligned}
x(t) \cos \left(-\pi k t^{2}\right) & =z(t) \cos \left(2 \pi f_{0} t+\pi k t^{2}\right) \cos \left(-\pi k t^{2}\right) \\
& =\frac{1}{2} z(t)\left(\cos \left(2 \pi f_{0} t\right)+\cos \left(2 \pi f_{0} t+2 \pi k t^{2}\right)\right)
\end{aligned}
$$

The $z(t)\left(\cos \left(2 \pi f_{0} t\right)\right.$ is the de-chirped signal (the de-chirped signal is a multiband signal) and it can be processed by the original MWC (note that a different reconstruction algorithm is adopted in our system). Here, we would like to explain why the MWC can only process multiband signal. As introduced in the previous section (see (7)), the number of spectral contents has an upper bound, but the number of spectral contents of chirp signal always exceeds the upper bound (see Fig. 4(a), the blue line indicates the occupied bandwidth and each number is the index number of one spectral content whose bandwidth is $B /\left(2 L_{0}+1\right)$ ), therefore, MWC is unable to deal with the chirp signal.

When the signal is de-chirped, (4) can be simplified as

$$
\boldsymbol{Y}(f)=\boldsymbol{A X}(f), f \in B
$$

where the $i$-th row vector of $\boldsymbol{Y}(f)$ is the discrete time Fourier transform (DTFT) of $y_{i}[n]$ and $\boldsymbol{X}(f)$ is the DTFT of $x(t)$. If the chirp rate is exactly estimated, $\boldsymbol{X}(f)$ is in the form of Fig. 4(b), which resides within a narrow frequency range (the blue line indicates the bandwidth of spectral contents). However, if the chirp rate is estimated with a small estimation error, $\boldsymbol{X}(f)$ is in the form of Fig. 4(c). In this case, the bandwidth of spectral contents is relatively wider (the purple line indicates the extra spectral contents compared with Fig. 4(b)). Therefore, we propose a modified 


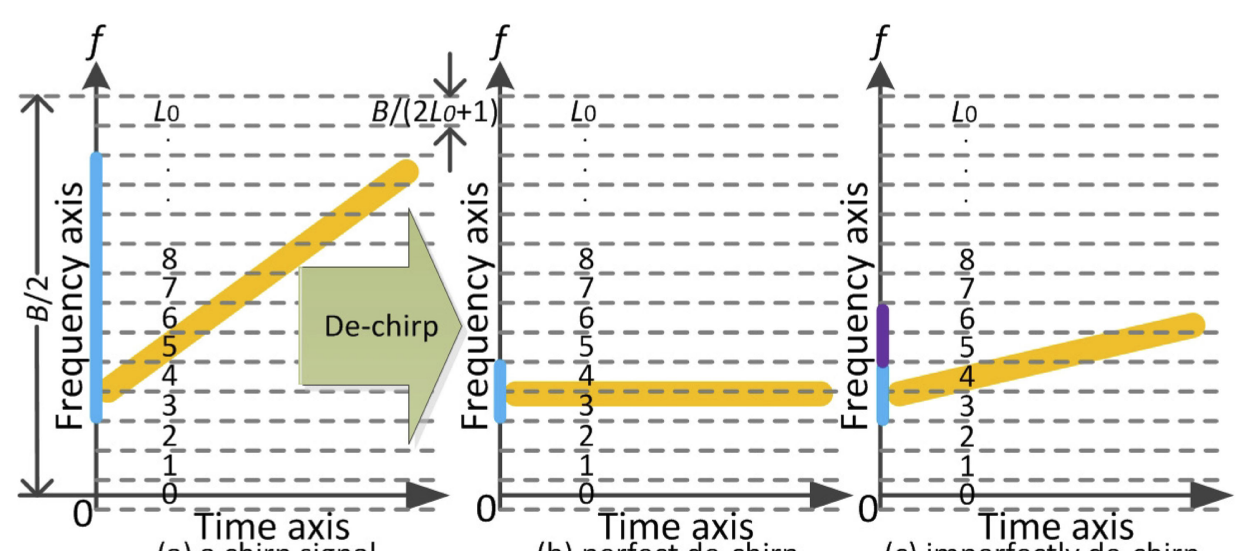

(a) a chirp signal

(c) imperfectly de-chirp

Fig. 4. Diagram of the de-chirp procedure. The target chirp signal is real signal, therefore, its spectrum has negative frequencies. In this figure, only positive frequencies are depicted. The bandwidth of positive part is $B / 2$; each number in this figure (i.e. $\left.0,1,2, \cdots, L_{0}\right)$ is the index for a certain frequency interval; the bandwidth of each sub-band is $B /\left(2 L_{0}+1\right)$.

version of the BMP algorithm $[16,17]$ to reconstruct the multiband signal, because the BMP can easily find these extra contents (for example, when the fourth content in Fig. 4(c) is estimated, the third and fifth contents can be easily found).

The signal reconstruction algorithm is designed for solving (11). However, since the row dimension of $\boldsymbol{A}$ (i.e. $m$ ) is smaller than its column dimension, (11) cannot be directly solved by finding the inversion of $\boldsymbol{A}$. We know that the dechirped signal only occupies a few frequency intervals, if the support set $S$ of occupied intervals is found $\left(S \subset\left\{-L_{0},-L_{0}+1, \cdots, 0,1, \cdots, L_{0}\right\}\right.$. Each entry of $S$ is called an index. An index indicates the location of one occupied interval, that is, it indicates the location of one spectrum content shown in Fig. 4), (11) can be simplified as

$$
\boldsymbol{Y}(f)=\boldsymbol{A}_{S} \boldsymbol{X}(f)
$$

where each column of $\boldsymbol{A}_{S}$ is chosen from $\boldsymbol{A}$ according to $S$ (here each entry of $S$ also indicates a column index of $\boldsymbol{A}$ ). Since $\boldsymbol{A}_{S}$ must has full column rank, it has

$$
\boldsymbol{X}(f)=\boldsymbol{A}_{S}^{\dagger} \boldsymbol{Y}(f)
$$

where $\uparrow$ means pseudo-inverse. Hence, when the support set is found, $\boldsymbol{X}(f)$ can be obtained. Nevertheless, finding the support set is still a tough task. In (11), $f$ is a continuous variable. As a result, it is hard to solve (11) [12]. To simplify the support set estimation process, the original MWC uses the continuous-to finite (CTF) module [12] which converts the continuous problem (11) into a finite problem (15). Note that there are many researches based on MWC, the CTF is essential for all of them; without the CTF, they cannot find the support set. The CTF first calculates the frame of $\boldsymbol{Y}(f)$, and any matrix $\boldsymbol{V}$ satisfies (14) is a frame.

$$
\boldsymbol{Q}=\boldsymbol{V} \boldsymbol{V}^{*}
$$

where $\boldsymbol{Q}=\sum_{-\infty}^{+\infty} \boldsymbol{y}[n] \boldsymbol{y}^{\prime}[n]$ (' denotes transpose) and $*$ denotes conjugate transpose. Then, solving (15) can find the support set of $\boldsymbol{X}(f)$, because the support set of $\boldsymbol{U}$ must be the same with that of $\boldsymbol{X}(f)$ [12] 


$$
V=A U
$$

The reconstruction algorithm (i.e. the support set estimation algorithm) is summarized in Table II, which is named multiple measurements block matching pursuit (MBMP). The algorithm adopts a stagewise estimation strategy, and in each stage a part of the support set can be found. It first calculates the correlation between $\boldsymbol{\Phi}$ and $\boldsymbol{r}$, then processes the preliminary test to estimate the location (i.e. the index number depicted in Fig. 4) of spectral contents. Here, $\operatorname{sum}(|\boldsymbol{C}|)$ returns a column vector whose $i$-th entry is the sum of absolute value of the $i$-th row of $\boldsymbol{C}$, $\operatorname{Max}(z, c)$ is the correlation maximization which returns indices of the $c$ biggest value of vector $z$ and $c$ is the estimation step size which determines how many indices can be estimated in one stage. The candidate list is a set in which all potential indices of spectral contents are gathered. The list consists of the estimated indices of the previous and current iteration and their adjacent indices (in Fig. 4, the third and fifth indices, namely these number 3 and 5, are adjacent indices of the fourth index; $\operatorname{block}(z)$ returns all adjacent indices of set $z$ ). Since spectral contents of the de-chirped signal are distributed in the form of blocks in frequency domain, gathering adjacent indices of estimated indices guarantees that all potential indices of spectral contents can be checked by the final test which outputs the estimation support set $F$ and residue $r$ of the current iteration. Until the iteration of the last stage is finished, the true support set is derived. Taking adjacent indices into account is the key that even if chirp rate is not exactly estimated, the support set of the imperfect de-chirped signal can still be estimated. When the support set $S$ is derived, then the digital version of original signal is $x[n]=\left[x_{1}[n], x_{2}[n], \cdots\right.$, $\left.x_{2 L_{0}+1}[n]\right]^{\prime}$, here, $x_{S}[n]=A_{S}^{\dagger} y[n] \cos \left(j \pi k n^{2}\right)$ (note that $k$ is chirp rate and $x_{i}[n]=0$, $i \notin S)$.

There are two differences between the MBMP and BMP. One is that MBMP is designed for estimating the support set of a matrix while BMP is for estimating the support set of a vector, therefore, there is an extra procedure of MBMP namely $\operatorname{sum}(|\boldsymbol{C}|)$. The other is that the halting condition of MBMP is adaptive and is derived during the decomposing of matrix $\boldsymbol{Q}$ while that of BMP is the $l_{2}$ norm of noise which has to be known in advance.

When decomposing $\boldsymbol{Q}$, we first calculate the eigenvalues and eigenvectors of $\boldsymbol{Q}$. Then, a matrix $\boldsymbol{V}$ which satisfies (14) can be obtained and each column of $\boldsymbol{V}$ is the eigenvector of $\boldsymbol{Q}$ multiplied by the square-root of its corresponding eigenvalue. Since the number of spectral contents has an upper bound $m / 2$ (when counting the conjugation of the signal), there is no need to collect more than $m / 2$ columns in $\boldsymbol{V}$, in other words, only $m / 2$ eigenvalues and their corresponding eigenvectors are necessary for calculating $\boldsymbol{V}$. However, in the presence of noise, the number of eigenvalues of $\boldsymbol{Q}$ is always bigger than $m / 2$ and we can utilize these extra eigenvalues and eigenvectors, which are actually related with the noise, to generate an adaptive halting condition. Then, we rewrite (14) in the following form

$$
\boldsymbol{Q}=\boldsymbol{V} \boldsymbol{V}^{*}+\boldsymbol{v} \boldsymbol{v}^{*}
$$

where $\boldsymbol{V}$ is produced by the $m / 2$ biggest eigenvalues of $\boldsymbol{Q}$ and their corresponding eigenvectors, $\boldsymbol{v}$ is produced by the rest of eigenvalues of $\boldsymbol{Q}$ and their corresponding eigenvectors. Assume the noise vector (i.e. samples of noise) is $\boldsymbol{e}[n]$, then it has, 
Table II. Pseudo-code of the MBMP

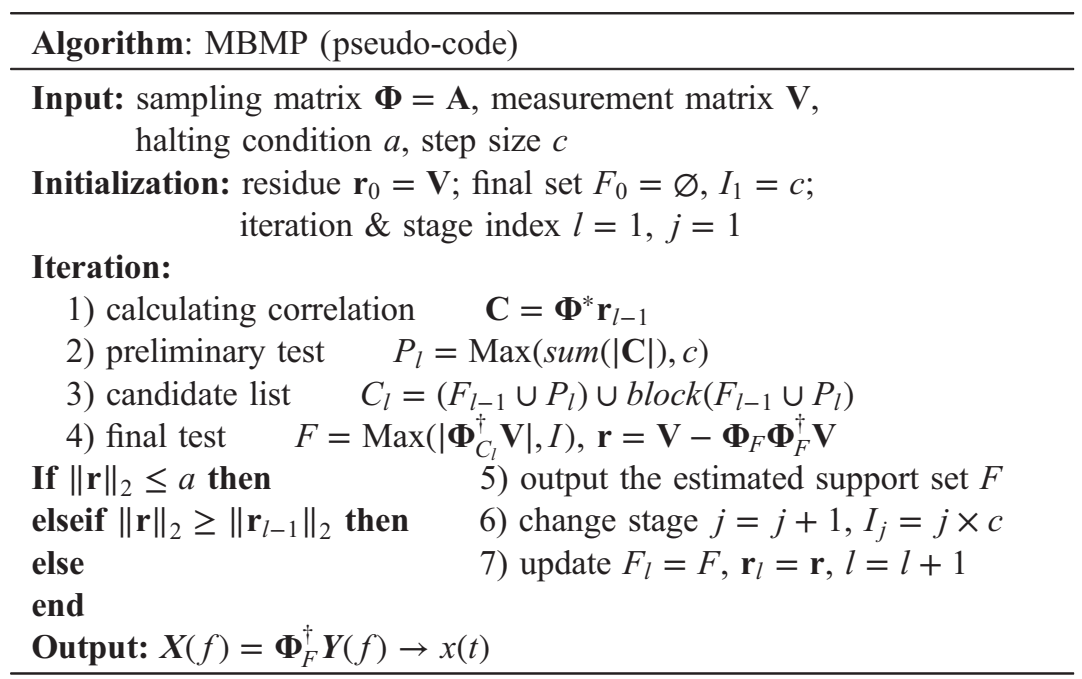

$$
\begin{aligned}
& Q_{1}=\boldsymbol{V} \boldsymbol{V}^{*} \\
& Q_{2}=\boldsymbol{v} \boldsymbol{v}^{*}
\end{aligned}
$$

where $\boldsymbol{Q}_{1}=\sum_{-\infty}^{+\infty}\left(\boldsymbol{y}[n]+\boldsymbol{e}_{1}[n]\right)\left(\boldsymbol{y}^{\prime}[n]+e_{1}^{\prime}[n]\right), \boldsymbol{Q}_{2}=\sum_{-\infty}^{+\infty} \boldsymbol{e}_{2}[n] \boldsymbol{e}_{2}^{\prime}[n], \boldsymbol{Q}=\boldsymbol{Q}_{1}+$ $\boldsymbol{Q}_{2}$ and $\boldsymbol{e}[n]=\boldsymbol{e}_{1}[n]+\boldsymbol{e}_{2}[n]$. Therefore, we can use the $l_{2}$ norm of $\boldsymbol{v}$ to estimate the noise floor of $\boldsymbol{V}$. When the most significant part of energy of $\boldsymbol{V}$ is captured by the algorithm, namely, when the residue $\boldsymbol{r}$ is only related with noise, the iteration should be halted. Therefore, the halting condition of the algorithm is

$$
a=c_{m}\|v\|_{2}
$$

where $c_{m}$ is a constant related with the number of sampling channels $m$. The relationship between $c_{m}$ and $m$ is analyzed, and the results are presented in Table III. This table is derived based on extensive simulations under several different signal noise ratio (SNR) levels (i.e. $0 \mathrm{~dB}, 10 \mathrm{~dB}, 20 \mathrm{~dB}$ ).

Table III. The relationship between $m$ and $c_{m}$

\begin{tabular}{|c|c|c|c|c|c|c|}
\hline$m$ & 50 & 60 & 70 & 80 & 90 & 100 \\
\hline$c_{m}$ & 2.3 & 2.5 & 2.7 & 2.9 & 3.1 & 3.3 \\
\hline
\end{tabular}

\section{Numerical simulations}

In this section, several experiments are carried out to verify our system is valid. The parameters of the system are set as follows: 1) $B=10 \mathrm{GHz}, 2) 1 / T_{s}=51.3 \mathrm{MHz}$, 3) $\left.\left(2 L_{0}+1\right)=195,4\right)$ time window is rectangle window and its width is $\left.1.97 \mathrm{us}, 5\right)$ $p_{i}(t)$ is the run length limited (RLL) sequence [15] and 6) the input signal is chirp signal $x(t)=z(t) \cos \left(2 \pi f_{0} t+\pi k t^{2}\right)(z(t)$ is the baseband signal whose bandwidth $b$ is randomly generated in each simulation, $b \in[1 \mathrm{MHz}, 51.3 \mathrm{MHz}], f_{0}$ is the carrier frequency which is also randomly generated in each simulation, $f_{0} \in[0, B / 2)$ ). In this section, each data in a figure is derived based on 500 times of simulations. 
In experiment 1, we investigate the performance of the chirp rate estimation algorithm under different number of time windows $w n$ and different chirp rate $k$. The simulation results are presented in Fig. 5 and in this experiment sampling channel $m=80$. In Fig. 5(a), the chirp rate of input signal is randomly generated. This figure shows when the number of time windows (i.e. $w n$ ) is bigger than 3 , the empirical probability of exact estimation is stable. The empirical probability equals the number of successful estimation divided by the total number of simulations. In Fig. 5(b), the number of time windows is fixed $(w n=3)$, and the $k_{\max }$ denotes the maximum chirp rate derived based on (9). This figure shows the algorithm can exactly estimate the chirp rate with a high probability when $k<k_{\max }$.

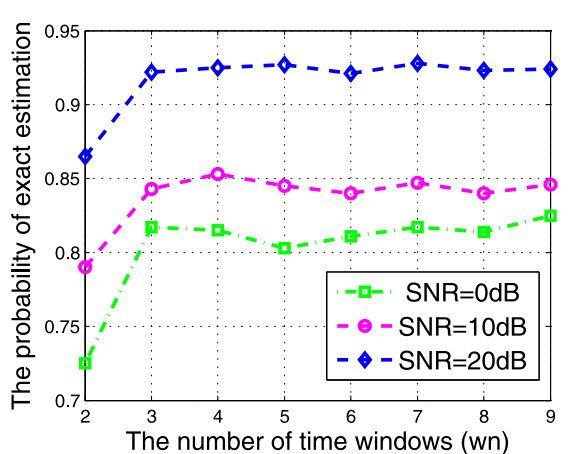

(a) Prob. of exact estimation vs. $w n$

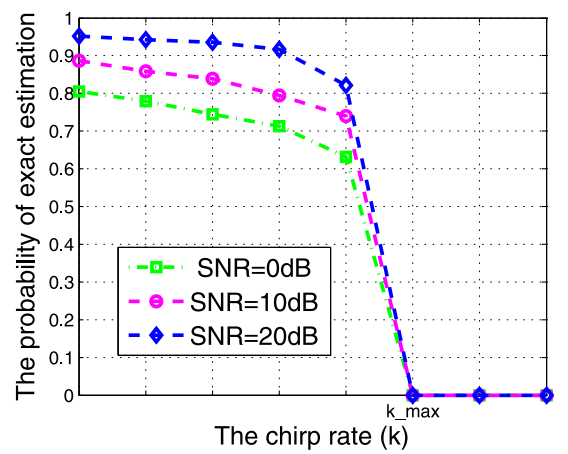

(b) Prob. of exact estimation vs. $k$

Fig. 5. Performance of the chirp rate estimation algorithm.

In experiment 2, we investigate the performance of the signal reconstruction algorithm under different sampling channels $m$ and different chirp rate $k$. In Fig. 6(a), it shows when the number of sampling channels gets bigger, the probability of successful reconstruction gets higher. And it also shows when SNR is high (i.e. $10 \mathrm{~dB} \& 20 \mathrm{~dB}$ ), the algorithm achieves an outstanding performance; when $\mathrm{SNR}=0 \mathrm{~dB}$ and the number of sampling channel is relatively bigger, the probability is about $83.5 \%$. In Fig. 6 (b), when $k \geq k_{\max }$, the algorithm cannot reconstruct the signal. This is because in this situation the chirp rate estimation algorithm fails to output the chirp rate $k$. Besides, we can see that probability curves in Fig. 6(b) are slightly higher than those curves in Fig. 5(b). This is because the MBMP algorithm is capable of reconstructing the imperfectly de-chirped signal.

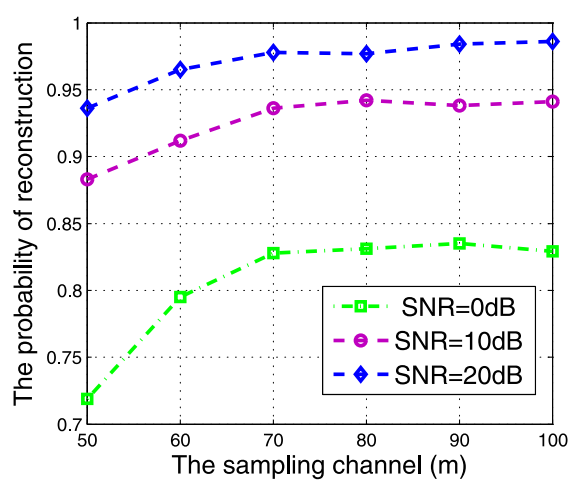

(a) Prob. of reconstruction vs. $m$

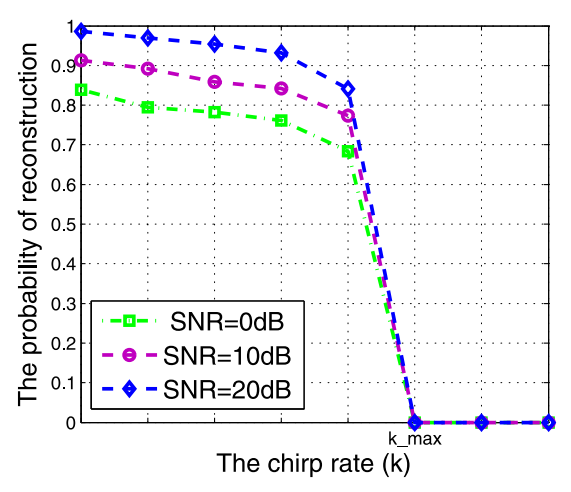

(b) Prob. of reconstruction vs. $k$

Fig. 6. Performance of the signal reconstruction algorithm. 
In experiment 3, the statistical normalized mean square error is analyzed, which is defined as $N M S E=E\left\{\|\hat{x}-x\|_{2}^{2} /\|x\|_{2}^{2}\right\}$ (here, $\hat{x}$ is the reconstructed signal, $x$ is the original signal, $E\{\cdot\}$ denotes expectation and $\|\cdot\|_{2}$ denotes the $l_{2}$ norm). According to Fig. 7, we can see that a sampling system with a bigger channel number has a smaller NMSE. However, it is impossible to build such a multitude of ADC channels in hardware. Fortunately, a solution is elaborated in [12], which can reduce the required hardware channels by increasing computational complexity.

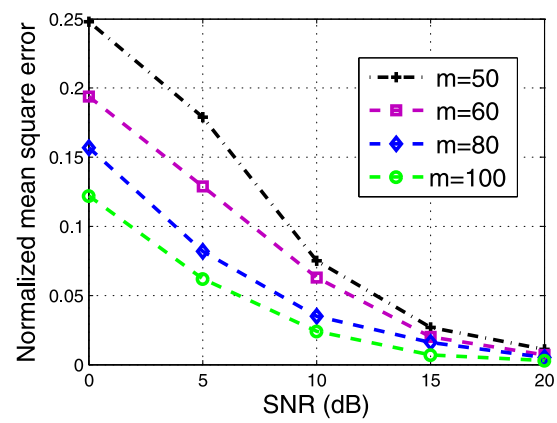

Fig. 7. The statistical normalized mean square error

\section{Conclusion}

In this letter, a novel compressive sampling system for acquiring chirp signal is proposed and analyzed. The most advantage of the proposed sampling system is that it does not require the prior information of carrier frequency and chirp rate. It first estimates the chirp rate of signal, then converts the signal into a multiband signal which can be reconstructed by the MBMP algorithm. Extensive simulation results confirm that our system is very appropriate for estimating the chirp rate and reconstructing the signal, and it achieves an outstanding reconstruction performance.

\section{Acknowledgments}

This work was supported by the National Natural Science Foundation of China under Grant No. 61601091. 